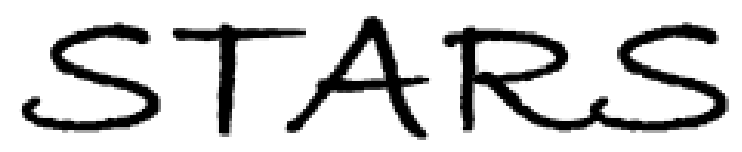

University of Central Florida

STARS

$1-1-2006$

\title{
Analysis and optimization of a Maglev system based on the Halbach magnet arrays
}

\author{
C. Ham \\ University of Central Florida \\ W. Ko \\ University of Central Florida \\ Q. Han \\ University of Central Florida
}

Find similar works at: https://stars.library.ucf.edu/facultybib2000

University of Central Florida Libraries http://library.ucf.edu

This Article is brought to you for free and open access by the Faculty Bibliography at STARS. It has been accepted for inclusion in Faculty Bibliography 2000 s by an authorized administrator of STARS. For more information, please contactSTARS@ucf.edu.

\section{Recommended Citation}

Ham, C.; Ko, W.; and Han, Q., "Analysis and optimization of a Maglev system based on the Halbach magnet arrays" (2006). Faculty Bibliography 2000s. 6198.

https://stars.library.ucf.edu/facultybib2000/6198

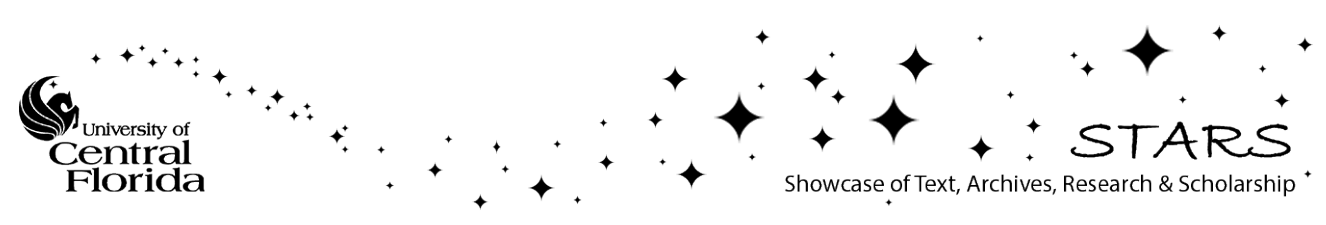




\section{Analysis and optimization of a Maglev system based on the Halbach magnet arrays}

Cite as: J. Appl. Phys. 99, 08P510 (2006); https://doi.org/10.1063/1.2162475

Published Online: 26 April 2006

C. Ham, W. Ko, and Q. Han

\section{ARTICLES YOU MAY BE INTERESTED IN}

Characteristic analysis of electrodynamic suspension device with permanent magnet Halbach array

Journal of Applied Physics 105, $07 A 314$ (2009); https://doi.org/10.1063/1.3068425

Forces on Moving Magnets due to Eddy Currents

Journal of Applied Physics 41, 2067 (1970); https://doi.org/10.1063/1.1659166

A new magnetic bearing using Halbach magnet arrays for a magnetic levitation stage

Review of Scientific Instruments 80, 045106 (2009); https://doi.org/10.1063/1.3116482

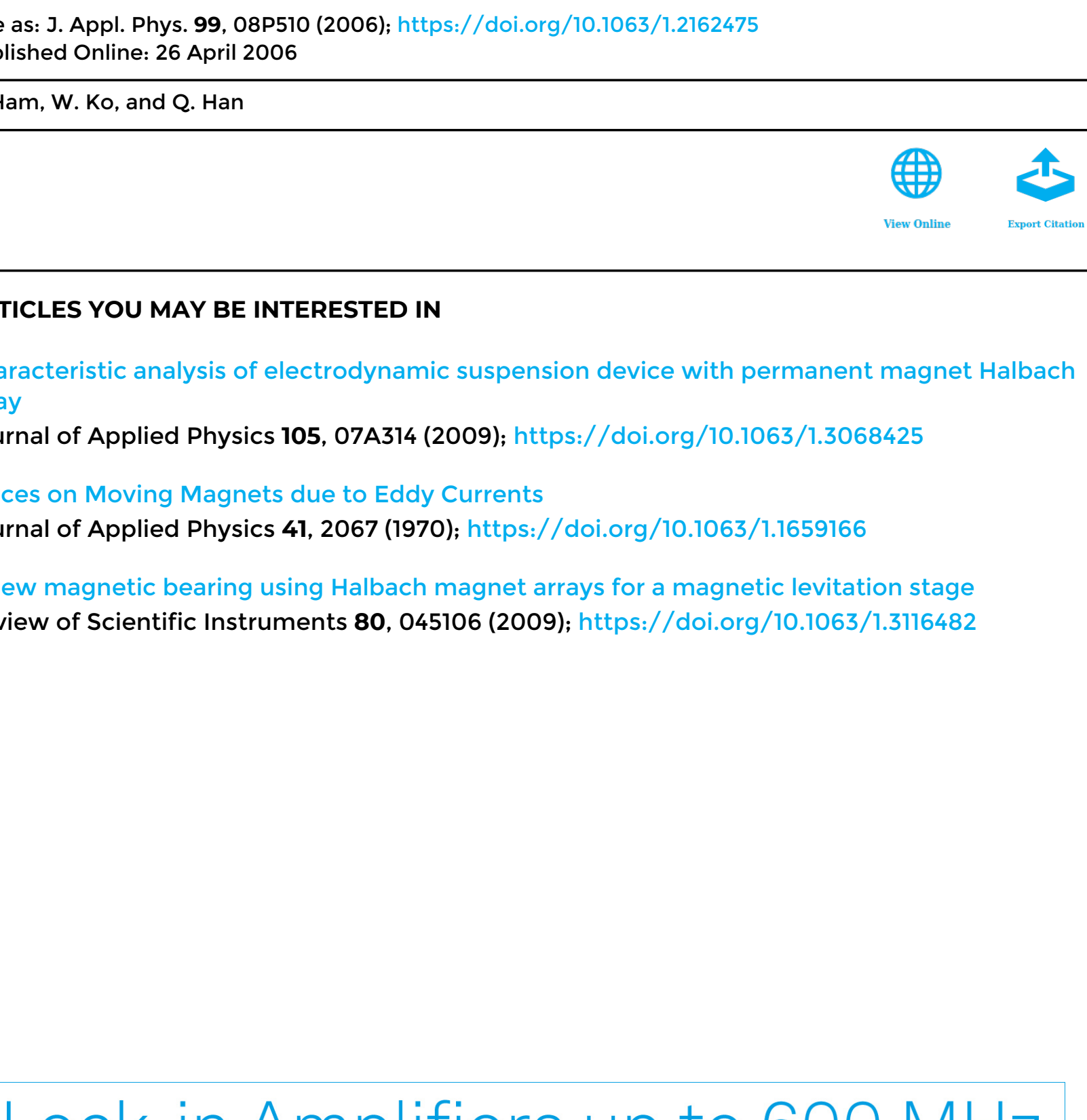

Lock-in Amplifiers up to $600 \mathrm{MHz}$

starting at $\$ 6,210$

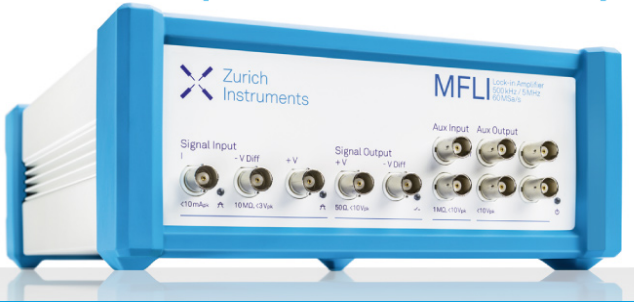

J. Appl. Phys. 99, 08P510 (2006); https://doi.org/10.1063/1.2162475

(c) 2006 American Institute of Physics.
99, 08P510 


\title{
Analysis and optimization of a Maglev system based on the Halbach magnet arrays
}

\author{
C. Ham, ${ }^{\text {a) }}$ W. Ko, and Q. Han \\ University of Central Florida, 12424 Research Parkway, Suite 488, Orlando, Florida 32826
}

(Presented on 3 November 2005; published online 26 April 2006)

\begin{abstract}
A systematic analysis and an optimization are presented for an electrodynamic suspension Maglev system based on the Halbach magnet arrays that promises to develop a feasible Maglev system. The magnetic field was modeled using scalar potential and Fourier series methods and then confirmed with the corresponding finite element method (FEM) result. The optimization of the array was derived based on the performance index defined as the ratio of the square of flux to the magnet weight of a unit volume. The study results were applied to the Maglev system at the University of Central Florida for measuring the current capability and optimizing the performance while gaining some insight into the system stability. (C) 2006 American Institute of Physics. [DOI: $10.1063 / 1.2162475]$
\end{abstract}

The Maglev system utilizes magnetic fields produced from ground-based electrical power sources to levitate the vehicle above the track. The Maglev enables a substantially lower vibrational and faster mass transportation trains. Maglev technology has also brought in a lot of interest in the space launch-assist concept since it may provide significantly improved safety, reduced cost, and reduced turnaround time to next launch for space missions.

In order to study the electrodynamic suspension (EDS) Maglev system, a small proof-of-concept model system was constructed by Lawrence Livermore National Lab that is currently located at the University of Central Florida. The Maglev track is a passive EDS magnetic levitation system employing special arrays of high-field permanent magnets, Halbach arrays, on the levitating cradle that is moving above a track consisting of a close-packed array of shorted coils. As shown in Fig. 1, the unique feature of the Halbach arrays is to produce a strong spatially periodic magnetic field on the front surface of the arrays, while canceling the field on their back surface. Relative motion between the Halbach arrays and the track coils induces currents in those coils. These currents levitate the cradle by interacting with the horizontal component of the magnetic field.

In order to analyze the existing Maglev track, first a Fourier series approach is used to model the magnetic field harmonics and then the outcomes are compared with finite element method (FEM) results for the validation of the proposed scheme. Figure 2 shows the geometry of a twodimensional permanent magnet (PM) sheet with a thickness of $d_{t}$.

For this configuration, the magnetization is written by

$$
\begin{aligned}
m=\mathbf{i} m_{x}+\mathbf{k} m_{z} & =\mathbf{i} \sum_{i} m_{x n}+\mathbf{k} \sum_{i} m_{z n} \\
& =\sum_{i}\left\{m_{0} f_{n}\left[\mathbf{i} \sin \left(k_{n} x\right)+\mathbf{k} \cos \left(k_{n} x\right)\right]\right\},
\end{aligned}
$$

where $m_{x n}$ and $m_{z n}$ are the horizontal and vertical compo-

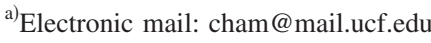

nents of the magnetization, respectively. Then, utilizing the superposition principle, the potentials are derived as ${ }^{1}$

$$
\begin{aligned}
& \varphi_{\text {canceled }}=0, \\
& \varphi_{\text {enhanced }}=\sum_{i} \frac{m_{0} f_{n}}{n k}\left(1-e^{n k d_{t}}\right) e^{n k z} \cos (n k x) .
\end{aligned}
$$

Equations in (2) can be solved using the boundary conditions. The potentials are continued at the boundary and go to zero at infinity. The flux density of the normal component is also continuous.

Figure 3 shows a four-piece Halbach array, with $\lambda=4 d$, $k=2 \pi / \lambda$, and thickness of $d_{t}$. Let us define $m_{z}(x)$ and $m_{x}(x)$ as the vertical and horizontal component of the magnetization, respectively. Then, the magnetization can be written as

$$
m_{z}(x)=m_{0} \Pi(4 x / \lambda) *\left(\sum_{-\infty}^{\infty}\{\delta(x-n \lambda)-\delta[x-(2 n-1) \lambda / 2]\}\right),
$$

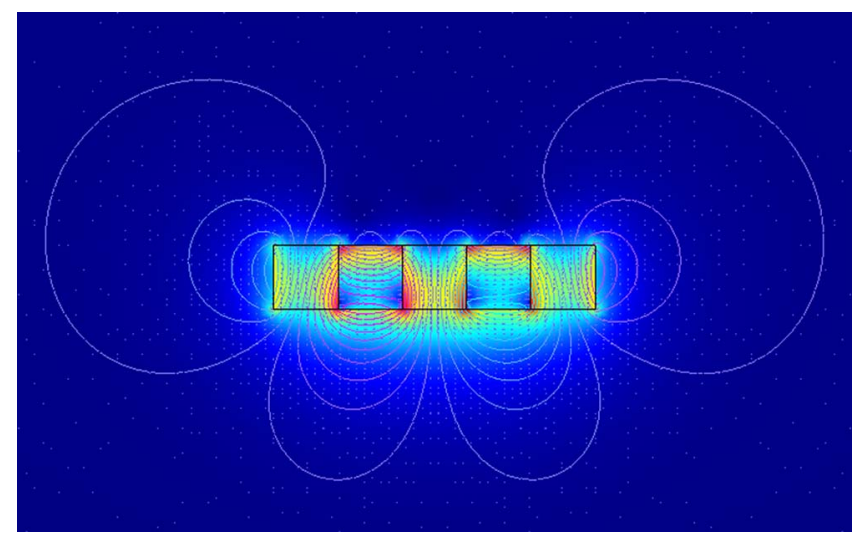

FIG. 1. (Color online) The horizontal field, $B_{x}(x)$, of a four-piece Halbach array. 


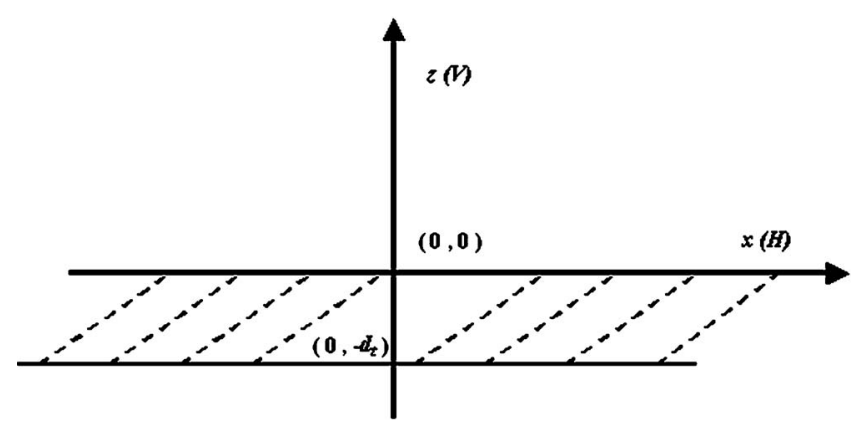

FIG. 2. The geometry of a PM sheet and coordinate definitions.

$$
\begin{aligned}
m_{x}(x)= & m_{0} \Pi(4 x / \lambda) *\left(\sum_{-\infty}^{\infty}\{\delta(x-n \lambda-\lambda / 4)\right. \\
& -\delta[x-(2 n-1) \lambda / 2-\lambda / 4]\}) .
\end{aligned}
$$

A periodic real valued power signal $x(t)$ with period $T_{0}$ can be represented by a Fourier series. ${ }^{2}$ Then, the Fourier series of $m_{z}(x)$ and $m_{x}(x)$ are given by

$$
\begin{aligned}
m_{z}(x)= & m_{0} \sum_{n=1}^{n=\infty} A_{n} \cos \left(2 \pi n f_{0} x\right) \\
= & m_{0} \sum_{n=1}^{n=\infty}-\frac{4}{\pi n}\left[\sin \left(\frac{\pi n}{2}\right) \cos \left(\frac{\pi n}{4}\right) \cos (\pi n)\right] \\
& \times \cos \left(2 \pi n f_{0} x\right), \\
m_{x}(x)= & m_{0} \sum_{n=1}^{n=\infty} B_{n} \sin \left(2 \pi n f_{0} x\right) \\
= & m_{0} \sum_{n=1}^{n=\infty}-\frac{4}{\pi n}\left[\sin \left(\frac{\pi n}{2}\right) \sin \left(\frac{\pi n}{4}\right) \cos (\pi n)\right] \\
& \times \sin \left(2 \pi n f_{0} x\right),
\end{aligned}
$$

where $m_{0}$ is the magnetization of the PM with remanent flux density $B_{r} . A_{n}$ is the $n$th harmonic amplitude of $m_{z}(x)$, and $B_{n}$ is the $n$th harmonic amplitude of $m_{x}(x)$. From (4) and (5), it
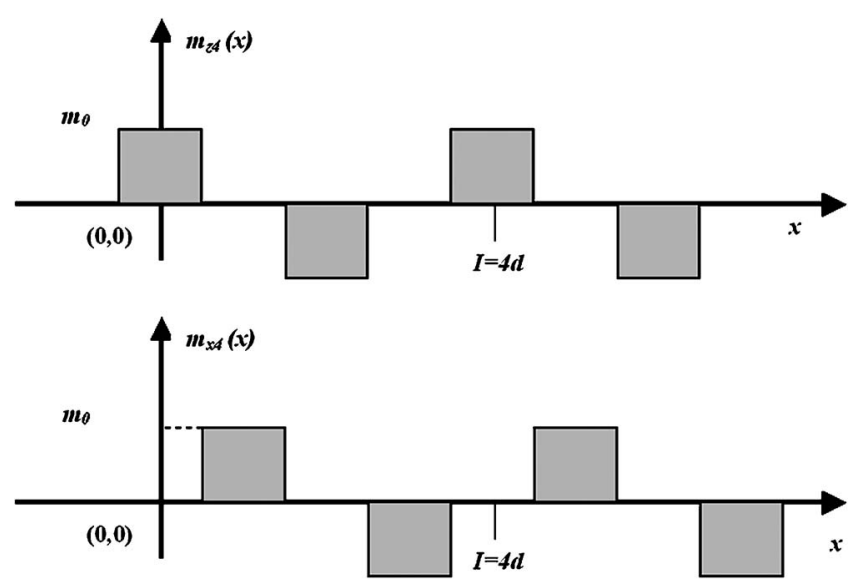

FIG. 3. The vertical (top) and horizontal (bottom) magnetization of a fourpiece Halbach array.

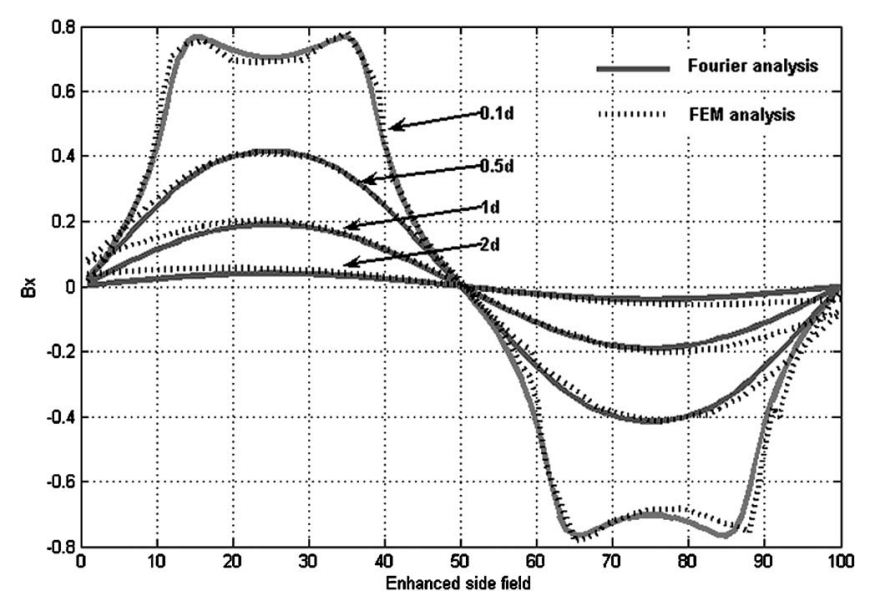

FIG. 4. $B_{x}(x)$, enhanced side at four distances for one period.

resulted in $A_{n}=B_{n}=0$ for $n=0,2 i, A_{n}=B_{n}$ for $n=4 i+1$ for the magnetic field on the enhanced side; and $A_{n}=-B_{n}$ for $n=4 i$ -1 for the magnetic field on the canceled side. Define $z$ as the distance from the observation point to the surface of the array. Then, the magnetic field intensity on the canceled side is given by

$$
\begin{aligned}
& H_{\text {canceled }} \\
& \qquad \sum_{n=3,7,11,15 \ldots(4 i-1) \ldots}\left[\mathbf{i} m_{0} A_{n}\left(1-e^{-i n k_{1} d_{t 4}}\right) e^{-n k_{1}|z|} \sin \left(n k_{1} x\right)\right. \\
& \left.\quad+\mathbf{k} m_{0} A_{n}\left(1-e^{-n k_{1} d_{t 4}}\right) e^{-n k_{1}|z|} \cos \left(n k_{1} x\right)\right] .
\end{aligned}
$$

And the magnetic field intensity on the enhanced side is given by

$$
\begin{aligned}
& H_{\text {enhanced }} \\
& \quad=\sum_{n=1,5,9,13 \ldots(4 i+1) \ldots}\left[\mathbf{i} m_{0} A_{n}\left(1-e^{-i n k_{1} d_{t 4}}\right) e^{-n k_{1}|z|} \sin \left(n k_{1} x\right)\right. \\
& \left.\quad+\mathbf{k} m_{0} A_{n}\left(1-e^{-n k_{1} d_{t 4}}\right) e^{-n k_{1}|z|} \cos \left(n k_{1} x\right)\right] .
\end{aligned}
$$

Note that in this paper only the analysis of the horizontal component, $B_{x}(x)$, is studied since the levitation is dependant on that component. ${ }^{3}$ While the vertical component can be utilized to provide the propulsion forces, it may be analyzed in a similar way.

The following analyses of the magnetic field flux densities shown in Fig. 3 are based on the four-piece square block Halbach arrays with $d=d_{t}$. The PM with $B_{r}=1.29 \mathrm{~T}$ and $\mu_{r}$ $=1.05$ is used in the calculation. Figure 4 shows the Halbach array magnetic field harmonics of the horizontal component on the enhanced side for four distances $(0.25 d, 0.5 d, d$, and $2.0 d)$ and the corresponding FEM results utilizing FEMLAB $^{\mathrm{TM}}$, which confirm the Fourier analysis technique. It is shown that beyond $0.5 d$, the magnetic field of the enhanced side is almost purely sinusoidal and the effect of high order harmonics has almost vanished.

The availability of an analytic theory of the Halbach array and its use in the Maglev track allows an analysis of the optimum parameters of the magnet system. In this section, the geometric optimization of the Halbach array only considers the levitation forces in which an optimized Halbach array provides maximum flux square over the magnet 


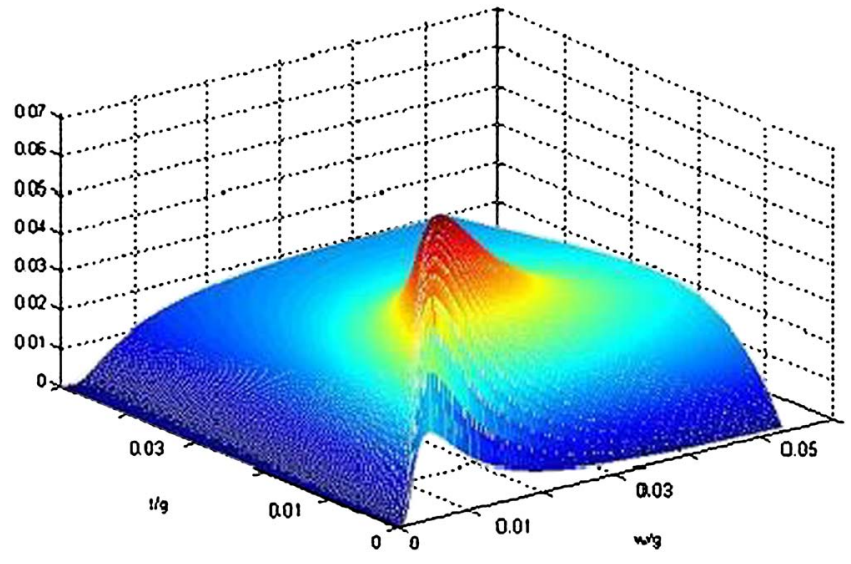

FIG. 5. (Color online) The optimization index.

weight per unit area. The optimization determines the magnetic block's horizontal length, vertical length, magnet array wavelength, and magnet thickness.

According to (8), the fundamental spatial flux density's amplitude at the enhanced side for the four-piece array is

$$
B=B_{r} A_{1}\left(1-e^{-k d_{t}}\right) e^{-k|z|} .
$$

It is obvious that the thickness $d_{t}$ is desired to be increased in order to maximize the magnetic field, but it also increases the magnet weight and cost. The optimization may be constrained by the magnet weight per unit area. While there are several approaches to optimize the magnet array's geometry, maximizing the flux squared over the magnet weight per unit area is used in this optimization study.

The following assumptions are used in the subsequent analyses: (1) the magnet array is large enough to consider it as infinite in length; (2) the edge effect is neglected; and (3) the optimization is based on a given nominal levitation height $z$. The optimization index is defined as

$$
\mathrm{Opt}=\frac{B^{2}}{W_{\mathrm{mag}}}=\frac{B^{2}}{\rho d_{t}},
$$

where $W_{\text {mag }}$ is the magnet's weight per unit area, and $\rho$ is the magnet weight for per unit area and per unit thickness. The array magnet thickness $d_{t}$ and the wavelength $\lambda=4 d$, where $d$ is the magnet block length, are optimized to get the maximum value of (10). Then, it follows that the fundamental spatial component optimization index is given by

$$
\mathrm{Opt}=\frac{B^{2}}{W_{\text {mag }}}=\frac{K^{2}}{\rho d_{t}}\left(1-e^{-2 \pi / \lambda d_{t}}\right)^{2} e^{-4 \pi / \lambda|z|}
$$

and the Opt function can be plotted as Fig. 5, which has a peak point.
The maximum point can be computed with (11) by differentiation that results in the optimized geometric parameter as

$$
d_{t}=\frac{4 \pi}{5}|z| \text { and } d=\pi|z| .
$$

Note that construction of the optimal rectangular block array requires two types of the magnet module while the square block array only needs one magnet module. So, considering the fabrication and cost reasons, the square-type Halbach array may be preferred in practical cases.

It is shown from the analyses that the existing track is not designed optimally to maximize its levitation capability. The thickness and length of the existing track's magnet are 1 and $2.5 \mathrm{~cm}$, respectively. So, utilizing (9)-(11) and $A_{1}$ $=0.9003$ the optimization index of the existing track is $\mathrm{Opt}_{E}=0.504$ that shows the levitation capability of the existing track is just half of the optimum case. It also shows that the existing track has $62.5 \%$ capability of the practical case that the Halbach array consists of four square magnet modules.

Also, from (9) the variation of $B$ with magnet thickness, $d_{t}$, can be determined. Since the weight of the magnets is proportional to $d_{t}$, an optimizing $d_{t}$ can be determined by differentiation that results in the optimum $d_{t}$ is equal to $0.2 \lambda$. Given the desired levitation height $z$, the wavelength that again optimizes the magnet weight is given by $\lambda_{\text {opt }}=4 \pi z$. The existing track is capable to levitate a mass 268 times heavier than its magnet weight for levitation height $z$ $=1 \mathrm{~cm}$ if all these optimization values are adopted into the design. These results will be utilized to the existing track's future enhancements.

A systematic analysis and an optimization have been presented for an EDS Maglev system based on the four-piece Halbach magnet arrays. The study focuses on analysis of the performance limits and optimization of the proof-of-concept system. The results show that the optimum performance of the existing track can be achieved when the array's magnet thickness and width are 2.5 and $3.1 \mathrm{~cm}$, respectively, where the desired levitation height is $1 \mathrm{~cm}$. The results of the study can be used as a technical framework for the field analysis and the performance optimization of future Maglev systems.

${ }^{1}$ D. Trumper, W. Kim, and M. Williams, IEEE Trans. Ind. Appl. 32, 371 (1996).

${ }^{2}$ R. E. Ziemer and W. H. Tranter, Principles of Communications (Wiley, New York, 1988).

${ }^{3}$ L. Tung, R. Post, and J. Martinez-Frias, Final Progress Report for the NASA Inductrack Model Rocket Launcher at the Lawrence Livermore National Laboratory, UCRL-ID-144455 (2001). 